Pecvnia, 9 (2009), pp. 85-109

\title{
Linguaggio e comunicazione degli organismi aziendali
}

Recibido: Julio 2009

Aceptado: Septiembre 2009

\author{
Giuseppe Catturi \\ catturi@unisi.it \\ Università degli Studi di Siena \\ Dipartimento di Studi Aziendale e Sociali \\ Piazza S. Francesco, n.8 \\ 53100 Siena (Italia)
}

Una metáfora eficaz para estudiar la empresa es considerarla "organismo viviente".

Este enfoque constituye la presuposición de la "teoría organicista", que estudia la empresa como un "sistema de relaciones" que es polo de intersección de "flujos comunicativos".

Las empresas comunican con lenguajes y signos distintos, en particular mediante: a) bienes y servicios; y b) datos contables.

Los productos tienen su propia semántica: describen y sintetizan historias, asuntos individuales y colectivos, e informan sobre los materiales utilizados y los procesos de fabricación, los entornos en que fueron creados o utilizados.

Los datos contables son síntoma y símbolo de la vida empresarial, porque son expresión sintética del conjunto de "actos de comunicación" de las empresas.

Desde esta perspectiva, las cuentas anuales "hablan" con acentos y tonalidad nuevas.
The metaphor of the "living organisms" is the most effective one to study organisations.

This approach, called "Organicist Theory", considers a firm as a net of relationships among the firm and the actors operating in its environment.

Firms communicate using different languages and signs. In our analysis, special attention will be paid to two firms' means of communication: a) goods/services; and b) accounting data.

Goods have their own semantics; they provide information on materials, technologies, processes, and contexts where they have been produced and used.

Accounting data are signs representing the firm's communicative actions. That Financial Statement starts talking with renewed meanings and tones.

In the Balance Sheet a forecast of the firm's future is provided by showing the 
El balance es la anticipación del futuro de la empresa porque expone la síntesis de la "potencialidad de energía" no expresada. La cuenta de "pérdidas y ganancias", quiere certificar el "reciente pasado" de la empresa.

Tenemos que dar énfasis al sistema de las relaciones empresa/entorno, y al Valor Añadido que es la grandeza que explicita esta dimensión.

El estudio de la empresa tiene que penetrar en su profundidad ético-motivadora hasta alcanzar lo más profundo de su "alma".

Palabras clave: Organismo, comunicación, dimensión, intereses, cultura, valor añadido. potentialities the firm has to use; the Profit and Loss Statement in its turn shows the recent past of a firm.

A strong emphasis has to be put both on the relationships between a firm and its environment and the Added Value as the quantitative measure able to represent such a dimension.

Business research work has to dig into ethical and behavioural meanings of firms in order to understand their "souls" fully.

Key words: Organism, Communication, Dimension, Interests, Culture, Added Value.

\section{INTRODUZIONE}

Considerare una qualunque azienda "organismo vivente" è, a mio parere, la più efficace ed esaustiva fra le molte similitudini e le variegate metafore che vengono utilizzate per avvicinare il ricercatore, od il semplice lettore, a quel concetto ed a quella entità socio-economica, intrattenendolo a riflettere tanto sulla sua struttura quanto sul suo modo di essere e di operare nell'ambiente in cui è inserita.

Del resto, un simile approccio costituisce il presupposto intuitivo di una teoria aziendale, appunto la teoria organicistica, che oramai si è universalmente diffusa, accompagnandosi alle altre conosciute da tempo. Si tratta, nello specifico, della teoria contrattualistica, con la più moderna teoria agenziale, e di quella sistemica, con la successiva teoria istituzionale, ognuna delle quali costituisce linea di studio e di ricerca che caratterizza orizzonti culturali affermatisi e condivisi ora qua, ora là negli ambienti del mondo scientifico - operativo ${ }^{1}$.

La teoria organicistica è oramai patrimonio degli studi aziendali e da qualche anno sta conquistando spazi e consensi sempre più ampi anche nell'ambiente scientifico anglo - sassone, oltre che, è naturale, in quello latino. Su di essa, infatti, si concentra l'attenzione e l'interesse degli aziendalisti che risultano attratti da una visione umanistica dell'attività economica per troppo tempo lasciata in balia di un "freddo" e "agnostico" tecnicismo. A tale riguardo si veda Stuart Kauffman (1995) At Home in the Universe, Oxford University Press; Ralph Stacey (1996) Complexity and Creativity in Organizations, Berrett Koheler; Gareth Morgan (1997) Images of Organization, Sage; A. De Geus (1997) The Living Company, Harvard Business School Press, Boston; Stephan Haeckel (1999) Adaptive Enterprise: Creating and Leading Sense-and-Respond Organizations. Foreword by Adrian Slywotzky. Boston: Harvard Business School Press; Richard T. Pascale et al. (2000) Surfing the Edge of Chaos, Texere. 
Frequentemente si utilizza la metafora del "vivente" anche per oggetti inanimati: è "viva" e "comunica", con un linguaggio proprio, un'opera d'arte ${ }^{2}$, un reperto archeologico, una foresta, una città, un'area territoriale, il nostro pianeta ${ }^{3}$, l'universo intero, etc., ma soprattutto una qualunque comunità di persone dai definiti confini culturali. Niente di strano, allora, se è organismo vivente l'azienda in virtù della comunità di persone che la caratterizza e la compone.

Attribuire a quell'entità socio - economica una simile valenza significa riconoscerla capace di interpretare i segni dei tempi e di sapersi adattare ai cambiamenti di scenario, superando indenne le turbolenze dei tempi storici che la sua traiettoria di vita impone ad essa di attraversare.

La costante prospettiva che adotto nelle mie ricerche aderisce completamente a tale orientamento culturale e in quell'ambito, è naturale, mi soffermo a riflettere sulle possibili configurazioni strutturali dell'azienda, ma anche sulla predisposizione di "barriere immunitarie", di "processi di omeostasi", di "valori etici", di "vizi", ecc., fino a riflettere sulle diverse dimensioni operative che possono essere individuate in ogni organismo aziendale ${ }^{4}$.

In questo studio enfatizzo la dimensione che definisco "politica" od "ambientale", la quale oggi appare di attualità sconcertante e di importanza indiscussa, aggiungendosi alla tradizionale dimensione "economica" ed a quella "sociale".

In relazione a tale obiettivo di analisi, intendo privilegiare lo studio dell'azienda come generatrice di un "sistema di relazioni", quale crocevia e polo di intersezione di "flussi comunicazionali" che la legano agli attori istituzionali operanti nell'ambiente in cui essa è presente ed alla Natura che la circonda, tanto che quelle relazioni costituiscono i suoi ricorrenti ed indispensabili "aneliti di vita".

2 "Un'opera d'arte è... anche un oggetto che viene investito di senso da un gruppo che intende comunicare, da una strategia di sottaciute intese relazionali. E il suo potere di dialogo attraverso lo spazio e il tempo trasfigura l'oggetto in un potente collante per la storia e la società, per gli individui e i luoghi". Massimo Kaufmann (2004) "Arte - domande e risposte", pag. 98, in Riscoprire il silenzio, a cura di Nicoletta Polla-Mattiot, Milano.

3 Cfr.: Fra i molti autori che condividono questa impostazione, ricordiamo Jim Lovelock, L'ipotesi Gaia, in P. Bunyard e E. Goldsmith (a cura di), L'ipotesi Gaia, pag. 59, Como 1992.

4 Giuseppe Catturi (a cura di) (2009) I vizi aziendali - Vademecun al contrario dell'azienda di successo, Ipsoa, Milano. 
E l'azienda è organismo vivente non solo perché comunica con i suoi simili, oppure perché domina e si adatta in modo dinamico alle modificazioni dell'ambiente in cui è immersa, attivando puntuali processi di omeostasi, od anche perché ha la capacità di memorizzare gli eventi che in modo diretto od indiretto la riguardano, ma soprattutto in quanto riferisce ed adegua i propri comportamenti ed il proprio modo di essere ad un "sistema di valori" che rappresentano veri e propri messaggi inscritti nel suo individuale DNA. Per questo motivo, l'azienda ha un'anima ${ }^{5}$.

Ognuna delle aziende che operano in un determinato periodo storico si muove nell"'orizzonte dei valori" ambientali di cui si impregna intimamente e, nello stesso tempo, è portatrice di "valori sintesi" condivisi e caratterizzanti la comunità di persone di cui è costituita. Qualunque azienda, infatti, vive "agganciata" alla storia di altri organismi socio economici e rapidamente muore se si interrompono le reciproche relazioni economiche, finanziarie o semplicemente di carattere informativo che sono espressione proprio del sistema dei valori ambientali accettati e condivisi.

Le mie riflessioni scientifico-operative aderiscono da sempre ad una simile interpretazione delle dinamiche socio-economiche e prendono avvio e si sviluppano basandosi su i due pilastri conoscitivi, fra loro correlati ed interdipendenti, che abbiamo appena rammentati: l'essere l'azienda organismo vivente e, in quanto tale, il mostrarsi capace di comunicare il proprio modo di essere all'ambiente circostante, ovvero alla pluralità indefinita dei soggetti che operano nella medesima comunità socioistituzionale, beneficiando insistentemente delle risorse che la prodiga Natura rende disponibili per attivare i processi tendenti alla creazione di valore economico.

\section{I PRESUPPOSTI CONCETTUALI AL LINGUAGGIO AZIENDALE}

Una qualunque azienda, al pari di ogni essere umano, ha dunque la facoltà di manifestare il proprio modo di essere e di operare nei confronti di quanti, in modo diretto od indiretto, si relazionano con essa. Ciò avviene in riferimento ad un suo individuale e particolare sistema di valori che si coniuga e si correla con quello dell'ambiente di riferimento, 
da cui sostanzialmente dipende. Quei valori, in definitiva, sono l'amalgama che rende possibile la costituzione in comunità dell'insieme delle persone che compongono l'unità aziendale e che la rendono viva.

La metafora più volte utilizzata -azienda/organismo viventeconsente di approcciare il tema del linguaggio e della comunicazione aziendale adottando i medesimi presupposti teorico-operativi normalmente considerati dagli antropologici e dai sociologi per quanto concerne le comunità umane.

È per questo motivo che riproponiamo brevemente alcune annotazioni poste su quella piattaforma culturale, senza aggiungere personalmente alcunché di innovativo. Tali annotazioni, pur nella loro essenzialità, appaiono comunque indispensabili ed esaustive per introdurre il lettore alla comprensione del fenomeno del linguaggio e della comunicazione propria degli organismi aziendali.

In effetti, è con l'esercizio della facoltà del linguaggio che l'uomo, per mezzo di suoni articolati, organizzati in parole, riesce ad individuare immagini, ad esprimere personali pensieri, sentimenti, visioni, attese, desideri ed a distinguere e selezionare i rapporti che instaura con i suoi simili, secondo convenzioni implicite che variano nel tempo e nello spazio, e con l'ambiente naturale che lo circonda ${ }^{6}$.

Nel proseguo della nostra indagine mostreremo come le prospettive di indagine appena evidenziate possano essere adottate anche per ogni singola azienda, poiché consentono di analizzare aspetti particolarmente significativi del loro relazionarsi con gli attori che operano nell'ambiente di riferimento.

Ci sembra utile spendere qualche parola sulla differenza, sicuramente nota, che è possibile riscontrare fra il linguaggio umano e quello degli animali. Anche quest'ultimi dispongono di linguaggi simbolici,

\footnotetext{
6 Nel linguaggio adottato da una determinata comunità di individui si possono considerare, allora, più elementi, ovvero:

- le parole che risultano dall'organizzazione di una molteplicità di suoni;

- le immagini, i pensieri, i sentimenti, i desideri, i rapporti con i simili e con l'ambiente naturale che vengono associati a quelle parole;

- le convenzioni, ovvero il sistema dei codici che trasmettono, conservano ed elaborano informazioni, consentendo di far comprendere, in modo identico, il significato delle parole formulate dagli individui che compongono una stessa comunità;

- la constatazione che il sistema di quelle convenzioni si modifichi nel tempo e risulti composto, in modo differente, nei variegati ambienti in cui viene adottato, risentendo, tale composizione, de tempi storici e dei tempi culturali vissuti dalle singole comunità sociali.
} 
ma mentre i loro sono connaturati, non si sviluppano e si riferiscono esclusivamente ad oggetti e ad eventi immediati e concreti, il linguaggio umano è in massima parte appreso, evolvendosi nel corso della vita dell'individuo e della comunità di appartenenza, e può riferirsi ad oggetti astratti.

Per questi motivi possiamo facilmente convenire come il linguaggio aziendale si avvicini molto più a quello umano che a quello animale, poiché, fra l'altro, esso si evolve nel corso della vita della stessa azienda che lo utilizza, adattandosi agli ambienti in cui l'azienda medesima viene generata e disegna la sua traiettoria di vita.

Insomma, il linguaggio è il modo particolare di esprimersi dei viventi, con riferimento ad un uso determinato della lingua di certi ambienti e di specifiche comunità di individui.

Questa affermazione richiede di soffermarsi sul concetto di lingua, cioè sul complesso di parole e locuzioni, ovvero sull'insieme di convenzioni necessarie per la comunicazione orale fra i singoli, consacrate dalla storia, dal prestigio degli autori che le hanno impiegate, dal consenso dei componenti della comunità che le utilizza per esprimere, comunicare, scambiare pensieri e sentimenti ${ }^{7}$.

Così, anche l'universo delle unità aziendali adotta una propria lingua, i cui caratteri sono definiti nel susseguirsi dei tempi storici e dagli ambienti culturali in cui quegli organismi sono generati e vengono consacrati autorevolmente dai soggetti, persone fisiche od organi che ne presiedono i comportamenti.

Gli elementi e gli aspetti che abbiamo appena evidenziato costituiscono lo specifico oggetto dell'indagine da cui attendiamo di rilevare interessanti correlazioni e similitudini fra il linguaggio aziendale e quello umano.

7 In senso più ampio, la lingua rappresenta il modo particolare in cui si esprime un determinato ambiente, sia esso sociale, artistico, scientifico, ma anche un singolo scrittore o poeta, nonché costituisce l'espressione di un particolare momento storico dell'evoluzione linguistica. 
3. ALCUNE RIFLESSIONI SUI CONCETTI DI INFORMAZIONE E DI COMUNICAZIONE

In senso del tutto generale, è informazione tutto ciò a cui l'uomo attribuisce un significato e che può essere utile per svolgere una qualunque attività. Da questo sintetico concetto emergono i caratteri dell'informazione, i quali, pertanto, possono essere riconosciuti:

a) nell'essere qualunque cosa a cui venga attribuito un senso, un significato di attesa, di speranza, di sentimento, di valore e, di conseguenza,

b) nel manifestare una utilità rispetto allo svolgimento di una determinata iniziativa.

Il concetto di informazione, tuttavia, può estendersi a tutto quanto venga trasmesso e recepito, ovvero comunicato ed accolto, che presenti una qualche utilità e funzionalità, più o meno immediata, in campo operativo.

Quest'ultima ottica interpretativa esalta con chiarezza l'aspetto dinamico insito nell'informazione; richiamando l'esistenza di

- un polo che la genera e di

- uno o più poli che la recepiscono.

Adottando una tale approccio di studio avviamo l'analisi al concetto di comunicazione. Essa, infatti, si caratterizza per due aspetti, il primo la considera come un trasferimento di informazioni codificate cioè di "segni" che sprimono o rappresentano stabilmente un dato oggetto fisico o mentale in base a certe regole - da un soggetto ad un altro, mediante processi bilaterali di emissione, trasmissione, ricezione ed interpretazione. L'altro aspetto della comunicazione consiste nel considerarla come una relazione sociale, mediante la quale due o più soggetti arrivano a condividere particolari significati.

Se ci poniamo sulla traiettoria del pensiero behaviorista, vi è ancora di più, poiché possiamo ritenere che ogni comportamento di un essere vivente che ne influenza un altro, in modo da provocare un comportamento rispondente, rappresenti una forma di comunicazione. Cosi, il comportamento di qualunque soggetto vivente, in definitiva, diventa un fatto di condizionamento per gli individui con i quali si relaziona.

Ne deriva che la comunicazione non solo 
- è la trasmissione di informazioni da un polo ad un altro, nel tentativo di far condividere a più soggetti determinati valori, ma

- è anche il tentativo di provocare un comportamento, ovvero di condizionare il modo di essere di coloro che sono investiti dalla stessa comunicazione.

Sono la condivisione di determinati valori e la conseguente uniformità di comportamenti, rese possibili dall'uso diffuso di un particolare linguaggio e di specifici segni, a condurre una qualunque aggregazione di individui, indipendentemente dal loro numero, alla realizzazione di una vera comunità sociale.

\section{L'ORGANISMO AZIENDA VIVE COMUNICANDO}

In relazione ed in conseguenza a quanto appena descritto si è oramai consolidato un filone di studio a carattere pluridisciplinare (che coinvolge la sociologia, la psicologia sociale, l'antropologia, la semiologia e perfino l'esegetica) relativo, da un lato, all'influenza che i processi di comunicazione hanno sulla struttura interna e sul comportamento di collettività comunque numerose, con particolare riguardo a quelle che si configurano come sistemi sociali e, dall'altro, al modo in cui i processi di comunicazione si strutturano, si evolvono e si differenziano, ossia vengono determinati dalla composizione e dai mutamenti interni ed esterni di una determinata collettività.

Le ricadute sull'azienda di un simile modo di concepire il concetto ed il processo di comunicazione emergono in tutta la loro significatività e la loro importanza operativa. È naturale che il rincorrersi delle modificazioni dell'ambiente in cui l'azienda vive ed opera, richiede continuamente la predisposizione di nuovi obiettivi operativi e di differenti criteri gestionali per consentire ad essa di mantenere nel tempo la sua finalità di fondo, cioè la soddisfazione dei bisogni umani, in via immediata o mediata, al grado più elevato possibile.

Le informazioni generate e trasmesse dall'azienda, talvolta qualitative, ma più spesso quantitative e quantitativo-monetarie, relative alle sue dinamiche gestionali, diventano, pertanto, oggetto di atti di comunicazione, la cui valenza semantica è compresa assicurando uniformità di manifestazioni e di modi di pensare fra la sorgente informativa e colui 
o coloro che ricevono il messaggio, siano essi attori economici che operano in un medesimo scenario od organi di una stessa azienda.

Il considerare l'organismo aziendale come comunità di persone, riunite funzionalmente in più organi, esalta allora l'importanza della cosiddetta comunicazione "interna". Spesso sono le difficoltà che emergono nel gestire il trasferimento di informazioni fra organi aziendali e soprattutto nell'interpretare in modo univoco, da parte dei vari responsabili di unità operative, i dati informativi trasmessi e ricevuti da quei medesimi organi, che creano tensioni e frizioni gestionali, costituenti causa non ultima del conseguimento di insoddisfacenti risultati economici e patrimoniali.

Alcuni di quei dati, opportunamente filtrati, cioè sottoposti ad una condivisa procedura di codifica, e tradotti in simboli comprensibili universalmente, travalicano il perimetro aziendale e si diffondono nell'ambiente circostante, diventando strumento efficace di comunicazione "esterna".

\section{NECESSITÀ DI COMUNICARE TRAMITE SIMBOLI}

Per trasmettere una qualunque informazione relativa al suo modo di essere ed al suo divenire nel tempo, ogni organismo aziendale deve trasformare quell'informazione in un segnale, cioè in un "dato", a carattere quantitativo o qualitativo, il quale diventa un vero e proprio simbolo di vita.

Tutte le aziende emettono una molteplicità di segnali, cioè di outputs informativi, che diffondono nel loro "intorno" socio - economico, permettendo di comunicare con gli organismi aziendali che vivono in quel medesimo ambiente e che, recependo il segnale trasmesso, entrano in contatto, cioè comunicano con l'azienda emittente.

Non c'è dubbio che le aziende, individui socio-economici, comunicano adottando molteplici linguaggi e segni. Per il fine delle nostra indagine, interessa la comunicazione che avviene fra le aziende che compongono l'universo degli attori ambientali e che si realizza mediante

- i beni fabbricati, i servizi resi e diffusi nell'ambiente ed

- i dati contabili. 


\section{IL BENE FABBRICATO OD IL SERVIZIO RESO SIMBOLO DI CULTURA AZIENDALE E MEZZO DI COMUNICAZIONE}

Uno dei "simboli" o "segni" fra i più importanti e significativi della cultura di una qualunque azienda è, senza alcun dubbio, il bene che essa fabbrica od il servizio che appresta.

Questi ultimi, in effetti, caratterizzano il momento storico della ininterrotta traiettoria di sviluppo disegnata dall'organismo aziendale e, quindi, della capacità che quello stesso organismo socio-economico manifesta nel vivere il suo tempo e finanche nell'interpretarne i segnali di cambiamento, ovvero nell'anticipare il dispiegarsi di quei medesimi tempi.

Ciò che intendiamo affermare è che il fabbricare, in un certo momento, un determinato bene, o l'apprestare un qualunque servizio è concreta espressione non solo del patrimonio delle conoscenze che via via si sono cumulate nei componenti della comunità di persone che compongono l'organismo aziendale, ma anche della tecnologia dei processi di creazione del valore adottati dall'azienda medesima e soprattutto, proprio quel bene o quel servizio è manifestazione del tentativo di interpretare il futuro da parte dell'unità aziendale che lo ha prodotto.

Se, infatti, la tecnologia impiegata nei processi di produzione può essere patrimonio di un numero indefinito di aziende, è il patrimonio delle conoscenze, delle abilità, ovvero delle professionalità espresse dalle persone delle comunità aziendali che le rende singolari, uniche e non clonabili.

In ogni azienda è peculiare la combinazione "uomo processo", la quale risulta irripetibile nel tempo e nello spazio. Per questo motivo, l'output di quei processi, ovvero il bene fabbricato od il servizio reso, caratterizza in modo indelebile l'azienda che lo ha prodotto e diffuso nell'ambiente, cioè immesso sul mercato.

D'altra parte, quei beni e quei servizi testimoniano le capacità innovative possedute dall'azienda e la sua attitudine ad interpretare il nuovo che si avvicina, quasi ad anticipare i tempi, sopravanzando la concorrenza e conquistando o sollecitando i favori dei consumatori.

In definitiva, tradizione ed innovazione sono le componenti delle traiettorie di sviluppo e non solo di crescita di una qualunque azienda ${ }^{8}$.

8 Riteniamo di dover differenziare il concetto di sviluppo da quello di crescita. Crescita, infatti, richiama il processo che si traduce nell'aumento, verificabile ad intervalli successivi 
Le considerazioni appena esposte aprono la riflessione ai fatti aziendali relativi alla gestione esterna, cioè a quelli che costituiscono eventi di comunicazione azienda/ambiente.

In effetti, la tipologia del "valore creato" o meglio "assemblato", la sua misura in termini monetari e la tecnologia di processo mediante la quale quel valore è stato realizzato all'interno dell'unità aziendale, sono l'estrinseca manifestazione della sua specifica cultura.

E' proprio in conseguenza delle riflessioni appena avanzate che due aziende, pur operando nel medesimo settore economico ed adottando le stesse tecnologie nell'attuazione del processo di lavorazione, sostengono differenti costi unitari, ma soprattutto riescono, in modo differente, a "collocare" la produzione realizzata ed a raggiungere $i$ potenziali consumatori. Il bene fabbricato ed il servizio reso sono veicolo di trasmissione dell'immagine e della cultura dell'azienda che li ha assemblati, sicché possiamo opportunamente e con facilità stabilire una forte ed immediata correlazione ed interdipendenza fra tipologia di output economico e cultura dell'azienda che l'ha generato.

Del resto, i beni posseggono una loro semantica; essi, mentre descrivono e sintetizzano storie, vicende individuali e collettive, informano dei materiali impiegati, degli strumenti adoperati e dei processi seguiti nel fabbricarli, degli ambienti in cui sono stati "creati" od utilizzati.

Quando un cliente/consumatore acquista sul mercato un qualunque bene o servizio, quell'atto esprime una sostanziale condivisione dei valori dell'azienda che rispettivamente l'ha fabbricato o reso e che si trovano sintetizzati proprio in quel particolare "contenitore", il cui valore

di tempo, di una specifica e particolare dimensione dell'oggetto o del fenomeno sottoposto ad analisi, di cui si apprezza e si conosce la composizione sistemica e, quindi, la sua morfologia.

Così, il fenomeno della crescita si può manifestare in modo asincrono nei molteplici componenti o grandezze del fenomeno o nelle componenti del sistema e con andamento differenziato nei tassi di incremento di alcune sue grandezze, tanto da evidenziare un aumento distorto e sbilenco dell'insieme analizzato.

Il concetto di sviluppo supera i limiti operativi della crescita che abbiamo appena evidenziato; il primo, infatti, è ben più complesso, coinvolgente ed unitario della crescita. Esso attiene e non solo ad una sua parte o dimensione, ma all'interezza dell'oggetto analizzato e mette in gioco non singole misure quantitative, ma anche parametri ed apprezzamenti di tipo qualitativo, risentendo comunque della correlazione fra i dati quantitativi relativi alle molteplici dimensioni o grandezze dell'oggetto analizzato, allorché vengono considerate contemporaneamente e in modo unitario.

Insomma, lo sviluppo richiama l'armonia nell'aumento coordinato e sincronico di tutta la composizione sistemica nei suoi molteplici aspetti e dimensioni, poiché guarda la totalità e l'unitarietà del fenomeno o dell'entità analizzata. 
economico incorporato è offerto per il soddisfacimento dei bisogni. Si tratta a tutti gli effetti di un vero e proprio "atto di comunicazione".

Così, è proprio la cultura aziendale che influenza le scelte relative non solo a "cosa", "dove" e "come" produrre, ma anche quelle che attengono le metodologie di proposta e di diffusione del valore assemblato; si tratta di decidere sul modo di essere dell'unità socio-economica e di come essa debba rapportarsi all'ambiente in cui è inserita.

Queste ultime osservazioni aprono l'indagine ad orizzonti di studio ancora più vasti, rispetto a quelli intravisti, poiché la correlazione che si viene a stabilire fra azienda ed ambiente, in sostanza, è confronto fra cultura aziendale e cultura antropologica od ambientale.

Scrivendo di ambiente intendiamo riferirci non solo al mero atto di vendita dei beni fabbricati e dei servizi resi alle unità aziendali consumatrici, ma anche al complesso delle motivazioni che rendono quei beni e quei servizi più o meno accetti alle persone che compongono la comunità sociale perché capaci di soddisfare, nella misura più elevata possibile, $i$ bisogni da esse avvertiti. Per questo riteniamo che i ricavi che l'azienda consegue con le vendite esprimano il "valore riconosciuto" dall'ambiente del valore economico che l'azienda dichiara di aver "assemblato".

\section{IL DATO CONTABILE COME SIMBOLO DI VITA E FATTORE DI} COMUNICAZIONE AZIENDALE

Fra $i$ dati generati in modo incessante da una qualunque azienda, riconosciamo quelli di origine contabile come $\mathrm{i}$ più carichi di significati, poiché rappresentano, in modo irrevocabile ed indiscutibile, la sintesi delle vicende che legano l'unità aziendale all'ambiente in cui è inserita ed opera 9 .

Si comprende facilmente, allora, l'affermazione secondo la quale il dato contabile risulti sintomo e simbolo di vita aziendale. Il suo carattere più evidente è l'istantaneità del suo verificarsi, agganciata com'è alla momentanea rappresentazione di un determinato stato. Per

9 Naturalmente, trattiamo dei dati relativi alla Contabilità Generale ed il presupposto di base delle nostre considerazioni è quello che tale sistema di scritture sia tenuto in modo corretto e veritiero. 
questo motivo, mentre occorre sommare situazioni verificabili istante dopo istante per delineare il tracciato di vita di un qualunque organismo, così occorre disporre di una serie di dati omogenei per rappresentare e per documentare le vicende longitudinali di qualsivoglia azienda.

I dati contabili, in effetti, assumono valenza simbolica del modo di essere dell'organismo aziendale; essi, cioè, costituiscono la manifestazione sintetica dei modelli organizzativi adottati, della tecnologia di produzione seguita nei processi di lavorazione, della professionalità dei componenti la comunità aziendale, della correlazione e della dipendenza fra i livelli di potere che si manifestano al suo interno, etc.; insomma, i dati contabili sono manifestazioni dei valori che costituiscono la cultura dell'azienda che li ha prodotti.

Quei dati sono rappresentativi, altresì, del modo di rapportarsi di un tale organismo all'ambiente di riferimento, cioè dei comportamenti che esso ha assunto nei confronti delle unità aziendali che operano nel medesimo ambiente e di come queste ultime accettino e condividano il valore assemblato dall'azienda considerata, acquistandone i beni fabbricati o godendone i servizi resi.

I dati di conto, dunque, sono espressione sintetica dell'insieme degli "atti di comunicazione", cioè delle relazioni che un qualunque organismo aziendale ha attivato con le altre aziende inserite nel medesimo tessuto socio-economico. Il dato contabile costituisce, come abbiamo già evidenziato, non solo la manifestazione quantitativo-monetaria di un evento della "storia" di un'azienda, quanto piuttosto la risultante di un sistema di valori che trovano concretezza numerica nel dato medesimo e che hanno costituito, da un lato, premessa e motivazione dei suoi comportamenti e, dall'altro, struttura e conformazione dell'ambiente in cui opera.

Del resto, i valori della cultura delle aziende sono sintesi e composizione delle prospettive, dei desideri, delle attese delle persone che costituiscono quelle comunità; così, l'interpretazione delle vicende gestionali, che si basano su dati quantitativo-monetari, irrevocabilmente acquisiti al sistema contabile, facilitano la prospettazione di orientamenti futuri e spingono l'analista a travalicare il dato momentaneo e contingente, proiettando l'azienda in orizzonti temporali più o meno lontani.

$\mathrm{Ne}$ deriva che a quei simboli si aggiunge la qualifica di contabili sia per il fatto di essere raccolti in modo omogeneo in specifici prospetti, tradizionalmente conosciuti come "conti", che per le operazioni 
di computo aritmetico che si possono effettuare nell'ambito di ogni singolo prospetto ${ }^{10}$.

Così, pur cambiando gli orizzonti culturali dell'ambiente e dell'azienda considerata, il conto ha continuato a dominare nel tempo la scena economica, proprio perché ha sempre avuto, e continua ad avere ancora oggi, il carattere di strumento di processo; esso, infatti, è il "luogo" in cui si cumulano, per mezzo di opportune annotazioni aritmetiche, eventi, fatti, situazioni, allorché questi vengono tradotti in simboli omogenei a carattere quantitativo o quantitativo-monetario.

Si avverte l'esigenza, pertanto, di risalire dal dato contabile alla cultura dell'azienda che l'ha prodotto, attraverso un'attività di introspezione scientifica che non avendo niente di meccanico si caratterizza per sensibilità ed intuizione personali.

In effetti, il contare in ambito aziendale non può limitarsi a tracciare vicende quantitativo-monetarie di patrimoni più o meno estesi, ma deve penetrare ancor più in profondità, rilevando e comunicando vicende di famiglie, di aziende, di comunità sociali, di popoli; cosicché il numero appare veramente sintesi di eventi storici e codice di situazioni esistenziali: abbiamo perso il gusto e la capacità di addentrarci nell'intima simbologia dei numeri e, quindi, nelle bellezze più profonde dell'animo umano e dei suoi comportamenti.

I traffici mercantili, il comprare ed il vendere, il produrre ed il consumare sono oramai diventati manifestazioni totalizzanti del viver quotidiano, provocando la ricerca esclusiva del dato quantitativomonetario e trascurando, invece, la comprensione dei valori ideali su cui si orientano i valori storici di qualunque comunità di individui.

Il modulo monetario, codice di trasformazione dei comportamenti aziendali in dati di conto, semplifica indubbiamente le determinazioni computistiche, ma massifica l'uomo, lo rende anonimo, ne appiattisce i valori, ne annebbia le storie, le motivazioni, gli interessi, le

10 Conto significa calcolo, misurazione ed anche enumerazione, ovvero operazione aritmetica attraverso la quale si valuta un totale attribuendo i numeri in ordine progressivo a persone, animali, cose; il conto, pertanto, inteso nel più largo senso è, essenzialmente, costituito da quantità (espresse in unità di valore o di misure fisiche) riguardanti un dato oggetto e insieme composte al fine di rilevare la variabile e commensurabile grandezza del medesimo. Il conto, in effetti, risponde al preciso bisogno di tener memoria di fatti o di eventi che hanno interessato uno specifico patrimonio durante un arco di tempo dato, mentre il suo scopo è quello di offrire informazioni utili a quanti devono decidere se continuare o rettificare le azioni amministrative intraprese. 
sconfitte, le vittorie... Occorre ritornare a far "parlare" i valori storici: i beni fabbricati, i servizi resi, i processi di lavorazione sviluppati, le strutture organizzative adottate devono enfatizzare l'uomo come attore e destinatario delle vicende universali ${ }^{11}$. Il modulo monetario, allora, espressione di quei numeri, non è più da considerarsi come "schiacciasassi del valore"; cosicché, il compito dell'analista che interpreta i documenti contabili di una qualunque azienda è quello di scavare sotto la scorza della superficie quantitativo-monetaria del dato di conto per apprezzare il mondo interiore dell'azienda medesima, cioè i suoi valori e la sua cultura.

Abbiamo perso la capacità di penetrare le profondità del numero e, quindi, del dato contabile, oramai privo degli aloni di trascendenza; la dimensione monetaria delle azioni a carattere economico ci costringe a valutazioni di convenienza, di opportunità e non tanto di sensibilità e di responsabilità umane.

Così, la storia aziendale descritta dai dati contabili non è solo storia di un singolo organismo socio-economico, ma della comunità degli uomini che la compongono e che ne esprimono la cultura. Per le intime e profonde correlazioni con l'ambiente di riferimento, la storia aziendale è anche la storia di tutte le persone che costituiscono la comunità sociale di appartenenza e dei propri orizzonti culturali; insomma, facendo storia aziendale facciamo storia sociale.

Se è comunemente accettato il fatto che l'attuale alfabeto, insieme con i numeri indio-arabi, sia stato strumento di intensa promozione dei traffici mercantili, agevolando la trasmissione di informazioni fra operatori economici, appare significativo, e forse anche originale, trattare del conto come mezzo di informazione e di comunicazione non solo fra addetti agli affari, ma anche nei confronti di coloro che comunque hanno interesse alla espressione sintetica dei rapporti di scambio. Così il conto, essendo capace di trasferire informazioni, assume rilevanza anche come strumento di trasmissione di cultura da un popolo ad un altro, poiché i modelli di comportamento, l'insieme delle credenze, dei valori, delle norme e dei presupposti che formano il substrato di ogni cultura trovano frequente traduzione in termini di conto. Ci sembra, infatti, che una qualunque comunità di individui possa sinteticamente apprezzare i caratteri

11 Giuseppe Catturi, Cor magis tibi Sena pandit, Intervento di apertura dei lavori al VII Incontro annuale di Business Ethics Research Centres in Europe, Siena, 22 marzo 1996. 
culturali di un'altra proprio dall'interpretazione di specifiche informazioni acquisibili dalle rilevazioni contabili ${ }^{12}$.

Ne deriva che gli storici aziendali possono trovare nel dato inserito nei prospetti di conto una fonte inesauribile di informazioni non solo dei valori della singola azienda che lo ha prodotto, ma dell'ambiente di riferimento e quindi della cultura antropologica riscontrabile nel periodo storico in cui collochiamo le dinamiche aziendali.

$\dot{E}$ in questo senso che possiamo affermare che il dato contabile è strumento di interpretazione dei segni dei tempi.

8. IL BILANCIO DI ESERCIZIO QUALE "LUOGO" DI INCONTRO/ SCONTRO DI CULTURE E POLO DI INTERSEZIONE DI FLUSSI RELAZIONALI

Mano a mano che $\mathrm{i}$ dati analitici vengono generati con la realizzazione delle operazioni di gestione, essi sono immediatamente riepilogati in documenti predisposti alla loro raccolta, in modo da poterli "trattare" mediante procedimenti matematico-statistici e giungere all'elaborazione di dati sintetici, ognuno rappresentativo di un particolare aspetto di vita aziendale.

Tali documenti si strutturano essenzialmente in prospetti, tradizionalmente denominati, come sappiamo, conti, i quali raccolgono sistematicamente i dati via via generati. Quei prospetti, infatti, risultano strumenti per rappresentare l'attività di un qualunque attore socio economico e per documentare gli atti di comunicazione con l'ambiente effettuati attraverso l'acquisto ed il successivo consumo di fattori destinati all'implementazione dei processi di creazione del valore attraverso la vendita dei beni fabbricati o l'apprestamento dei servizi.

L'interpretazione dei dati che emergono dai documenti contabili di sintesi dei fatti amministrativi, che una qualunque azienda predispone al termine di un determinato periodo di tempo, deve essere posta, tuttavia, in una dimensione un po' diversa rispetto a quella attribuita dalla tradizione degli studi aziendali, tanto che la loro attenta "lettura" evoca nuove sensazioni e provoca differenti risonanze, esaltando

12 Sull'argomento si veda ampiamente di Giuseppe Catturi (1987) La scrittura in partita doppia come mezzo di comunicazione tra operatori economici di differenti aree territoriali, in Aa.Vv., "Saggi di Economia Aziendale per Lino Azzini", pag. 249 e segg., Milano. 
interessanti associazioni di idee e ponendo pressanti interrogativi da cui si possono trarre altrettante risposte e specifiche motivazioni ad operare.

Le diverse strutture formali che possono essere assegnate ai documenti contabili di sintesi non rispondono, allora, esclusivamente ad esigenze di aggiornamento nella meccanica riepilogativa dei dati via via generati dall'azienda, ma piuttosto ognuna di esse tende ad esaltare un particolare aspetto della vita "interiore" e delle manifestazioni di "vita sociale" di quell'organismo socio-economico, perennemente mutevole nelle forme e nei comportamenti.

Dobbiamo convincerci che la strumentazione contabile ed $i$ documenti che periodicamente vengono redatti in termini quantitativomonetari intendono comunicare il "modo di essere" dell'organismo aziendale cui si riferiscono, cioè rendere noti i criteri seguiti sia nell'attuazione dei processi interni di creazione del valore che di quelli inerenti la sua diffusione nell'ambiente circostante.

Per questi motivi, il tradizionale Bilancio di esercizio risulta la rappresentazione di un "presente proiettato nel futuro con un inevitabile ancoraggio nel passato". Il tutto viene testimoniato non solo dalla composizione e dalle risultanze finali del Conto Economico, ma anche dall'entità degli stock riuniti nella Situazione Patrimoniale, la cui disponibilità all'utilizzo nei processi di produzione discende da operazioni di gestione che hanno oramai caratterizzato la storia dell'azienda.

In quest'ottica interpretativa, allora, quel documento, cioè il Bilancio di esercizio, perde molto del suo linguaggio usuale e comincia "a parlare" a chi lo sa intendere con tonalità e cadenze nuove, descrivendo vicende storiche vissute, comunicando, a chi lo sa leggere, comportamenti assunti, obiettivi gestionali raggiunti o solo ipotizzati od attesi. I numeri, di cui ai valori quantitativo-monetari inscritti nei prospetti di bilancio, assumono in pieno la valenza di simboli di un linguaggio che vuole essere comprensibile non solo a degli iniziati, ma anche nei confronti di tutti coloro che intendono raggiungere la conoscenza piena delle dinamiche aziendali.

Quel documento, in effetti, può essere analizzato sotto diversi angoli visuali, ovvero secondo differenti prospettive di indagine e per ognuna di esse emergono valenze interpretative di una realtà composita, ma certamente unitaria, che intendono rappresentare le meccaniche interne di formazione del valore e dei continui rapporti di scambio, cioè 
dei flussi comunicazionali, che si instaurano fra l'azienda e l'ambiente nel quale essa è inserita ${ }^{13}$.

A ben guardare, il Bilancio di esercizio è una tavolozza di "colori", ognuno dei quali vela il sottostante, creando l'effetto delle vicende storiche nel tempo.

Nella Situazione Patrimoniale, in effetti, risulta descritta l'anticipazione del "futuro" dell'azienda. Quel documento, in realtà, espone la sintesi delle "potenzialità di energia" ancora inespresse, ma disponibili nel momento della redazione del documento. Ogni elemento patrimoniale, pertanto, rappresenta una fonte da cui l'azienda medesima potrà attingere valore economico o finanziario in un futuro più o meno prossimo.

Il Conto Economico, invece, vuole certificare il "recente passato" vissuto dall'azienda; tale documento, infatti, documenta il valore economico goduto o consumato - $i$ costi di competenza- e quello diffuso - $i$ ricavi di competenza- nel periodo amministrativo appena trascorso.

Ma il Conto Economico che la tradizione della dottrina contabile ci ha fatto conoscere ed interpretare, indipendentemente dalla sua rappresentazione formale, risente di posizioni culturali che riteniamo oramai superate. A nostro parere, occorre abbandonare la ristretta ottica del proprietario ed orientarsi, invece, a quella dell'azienda considerata come organismo unitario inserito in un determinato ambiente.

In effetti, l'interesse del soggetto proprietario non è l'unico, né il prevalente, a cui riferire i comportamenti aziendali e le grandezze gestionali di sintesi; cosicché all'azienda riconosciamo, oltre alla sua naturale dimensione economica, anche una dimensione sociale ed una politicoambientale. Il tempo che viviamo è caratterizzato dall'enfasi che viene posta in particolare a quest'ultima dimensione aziendale che privilegia il sistema delle relazioni azienda/ambiente, ed è il "Valore Aggiunto" la grandezza gestionale di sintesi che meglio di ogni altra esplicita la dimensione politica dell'azienda, senza abbandonare quella sociale, ovvero l'ottica dell'azienda come comunità di persone, e finanche la tradizionale dimensione economica, allorché la si consideri strumento di generazione di profitto ad esclusivo vantaggio del proprietario.

13 Ciò che vogliamo far intendere è simile al comportamento di un qualunque prisma che riflette colori diversi a seconda dell'angolo di incidenza del raggio di luce che lo colpisce. 
Per proseguire nelle riflessioni appena delineate occorre rammentare brevemente la procedura di determinazione contabile del Valore Aggiunto.

Il montante di tale grandezza gestionale si ottiene, in via computistica, deducendo dal totale dei ricavi conseguiti dall'azienda nel periodo considerato, che rappresentano il "valore riconosciuto" dall'ambiente del "valore assemblato" e "diffuso" nell'ambiente medesimo dalla stessa azienda, l'ammontare dei "consumi intermedi", ovvero il valore economico prelevato dai "pacchetti patrimoniali di attivazione" come risulta nella seguente figura:

Figura 1

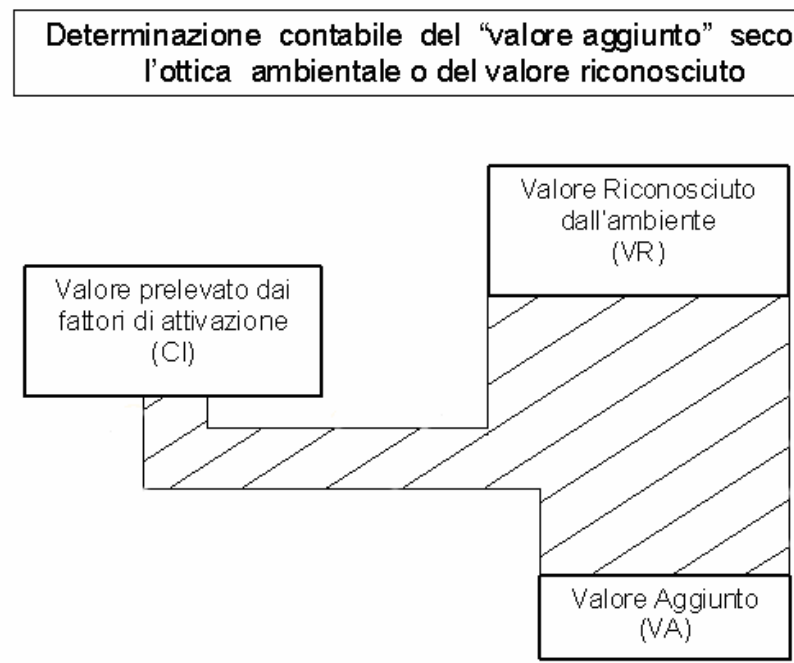

Proprio perché confortati dalla struttura formale del conto del Valore Aggiunto, possiamo penetrare più in profondità il contenuto sostanziale di quella grandezza, aprendo le nostre riflessioni a dimensioni interpretative che travalicano il tradizionale accertamento del risultato economico conseguito nell'arco di tempo considerato.

In tale prospetto di conto, infatti, si individuano facilmente, anche in modo formale, due aree operative: nella parte alta del prospetto, l'area "esterna" all'azienda e nella parte bassa quella "interna". Il significato di questi due ambiti si carica di conseguenze culturali ed operative di straordinaria rilevanza. 
In effetti, l'area esterna, cioè la parte del prospetto costituita dal Valore Riconosciuto dall'ambiente, comprende e rappresenta la sintesi del sistema delle relazioni e dei flussi comunicazionali che l'azienda instaura con gli attori operanti nel medesimo ambiente in cui è inserita. $\grave{E}$ evidente che la tipologia e le modalità di esecuzione di quelle relazioni rappresentano ed esprimono i caratteri della cosiddetta cultura antropologica ed è quanto sintetizziamo nello schema che segue:

Figura 2: Area "esterna" dell'azienda

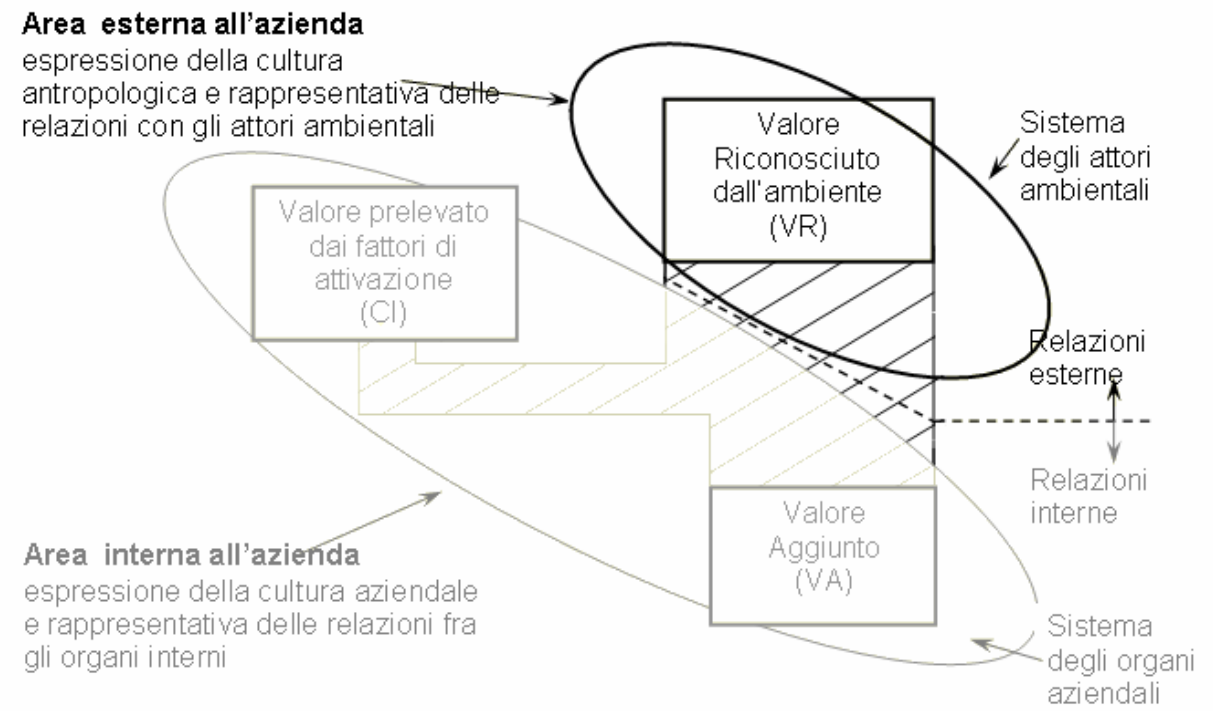

L'area interna, invece, resa evidente dalla parte bassa del prospetto di conto che mostra $i$ valori consumati sia dei fattori patrimoniali di attivazione (o Consumi Intermedi) che di quelli durevoli (da cui la composizione del Valore Aggiunto), vuol rappresentare l'aggregato delle relazioni generatrici di flussi di natura economica che si instaurano nell'ambito del sistema degli organi dell'azienda e, quindi, sono espressione forte e significativa della sua cultura.

Le considerazioni appena espresse, allora, vengono rese evidenti nel seguente schema: 
Figura 3: Area "interna" dell'azienda

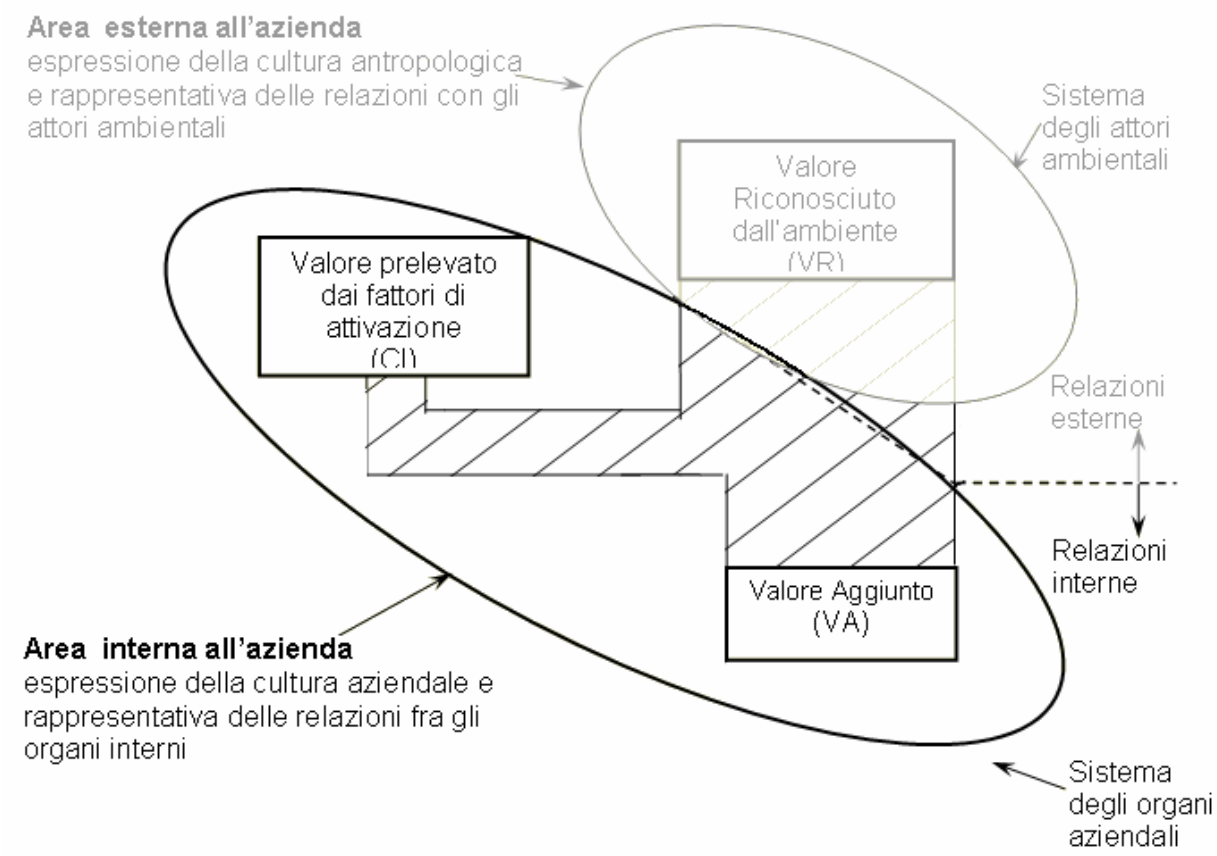

La rappresentazione grafica del conto del Valore Aggiunto e soprattutto le considerazioni a carattere teorico-operative che quella rappresentazione ha permesso di fare, consentono di puntualizzare l'importanza della dimensione politica disegnata da una qualunque azienda. Quell'orizzonte dimensionale, in una visione centripeta dell'attività esercitata dall'unità aziendale, è reso esplicito proprio dall'area esterna al perimetro di tale organismo socio-economico, la quale, pertanto, comprende l'insieme delle relazioni che l'azienda medesima instaura con l'universo degli attori ambientali.

La dimensione più strettamente sociale, a carattere centripeto, viene individuata, invece, dall'area interna al perimetro dell'azienda considerata. In tale area vengono comprese e sintetizzate le relazioni che si instaurano fra gli organi di quella stessa azienda e le azioni che essi compiono sugli elementi del patrimonio disponibile per realizzare il processo di produzione che la caratterizza.

Nel conto del Valore Aggiunto, allora, si rilevano con immediatezza ed appaiono in tutta evidenza le correlazioni e le 
interdipendenze azienda/ambiente, evidenziando l'incontro/scontro fra la cultura ambientale od antropologica e la cultura aziendale.

Approfondendo questa direttrice di indagine è facile prospettare considerazioni di tipo etico. Del resto, i comportamenti dei soggetti, individuali o collettivi, si orientano proprio in riferimento ad un modello costituito da una determinata e condivisa griglia di valori etici, la quale costituisce e caratterizza il nucleo centrale degli orizzonti culturali.

Per avvalorare le sensazioni appena esposte basta riflettere sul fatto che il gradimento od il rifiuto di certi beni o servizi, che risultano disponibili sul mercato, da parte di un numero più o meno elevato di clienti/consumatori, dipende indubbiamente dal loro sistema di valori e, quindi, dalla loro cultura. Cosi, la fabbricazione di quei medesimi beni o l'erogazione di quei servizi, su iniziativa di una qualunque azienda, si correla immediatamente al sistema dei valori etici che costituisce la piattaforma sulla quale quella medesima azienda fonda la propria attività ed esprime il proprio modo di essere e, quindi, la propria cultura.

I consumatori, spinti da motivazioni etico-culturali, rifiutano, in modo sempre più massiccio, l'acquisto di determinati prodotti immessi sul mercato da una certa azienda, perché nocivi alla salute o perché impiegano con violenza giovani risorse umane o perché il fabbricarli richiede l'adozione di processi tecnici di lavorazione che implicano la distruzione di risorse naturali non rinnovabili o la produzione di residui o di rifiuti non riciclabili. Un loro simile comportamento si traduce, così, per l'azienda, nel conseguimento di un minor ammontare di ricavi rispetto a quello possibile e, pertanto, in un minor "riconoscimento di valore" da parte della comunità degli attori socio-economici, rispetto a quello che l'unità aziendale medesima dichiarava di aver creato e di cui attendeva il riconoscimento; insomma, non serve fabbricare beni che la cultura antropologica rifiuta ${ }^{14}$.

Il conseguimento del profitto, qualunque ne sia l'ammontare, in definitiva ed in concreto, testimonia l'apprezzamento della specifica cultura aziendale da parte della comunità sociale; viceversa, il sostenimento di perdite rende palese l'esistenza di un gap fra il modo di essere

14 La situazione esposta nel testo presuppone uno scenario socio - economico in cui tutti gli attori risultano informati in modo sufficiente, sollecito e veritiero. In realtà, è proprio tramite l'informazione distorta o non corretta che è possibile "drogare" il mercato ed indurre alcune aziende a comportamenti che possono sortire effetti dannosi per i componenti della propria comunità e di quella, più vasta, sociale. 
dell'unità aziendale e le attese della collettività. Si tratta, in pratica, di una crisi di rigetto: la perdita subita, infatti, dimostra che è in atto un processo di rifiuto da parte dell'organismo sociale nei confronti di un suo organo, quella particolare azienda, i cui comportamenti ed attività non risultano coerenti e armoniosamente coordinati con il tutto ${ }^{15}$.

È sicuramente interessante ritornare al prospetto di conto del Valore Aggiunto redatto al termine del periodo amministrativo, poiché in esso si possono intravedere aspetti relativi alla "psicologia" dell'azienda che lo ha redatto ed alla sua "sociologia". Il senso di una simile affermazione si percepisce osservando ancora la struttura formale di quel prospetto. Il suo settore (area) sottostante, infatti, vuole mostrare le dinamiche gestionali verificatesi allinterno del perimetro aziendale, rendendo conto dei flussi economici sviluppati dal sistema delle relazioni che si sono instaurate fra gli organi aziendali e relativi ai consumi degli elementi patrimoniali impiegati nella realizzazione dei processi di produzione. Il settore sovrastante del medesimo prospetto, invece, quello che rende conto dei ricavi conseguiti documenta l'intensità dei flussi economici generati dall'insieme delle relazioni che l'azienda ha instaurato con gli altri attori operanti nell'ambiente di riferimento.

Sono questi i motivi che ci spingono a diventare più esigenti nell'attribuzione di significati e di valori ai numeri che costituiscono il nostro linguaggio più usuale ed il nostro mezzo privilegiato di comunicazione.

È su questa stessa linea di pensiero che possiamo sviluppare l'indagine concernente la ricerca della più efficace ed efficiente metodologia di inserimento di modelli d'azienda e di criteri gestionali in aree culturali differenti da quelle in cui quei medesimi modelli e criteri hanno avuto origine e si sono affermati.

La stessa direttrice di analisi consente di individuare le motivazioni che consentono ad un'azienda di conseguire positivi risultati economici in determinate aree territoriali e contemporaneamente subire delle perdite in aree diverse, pur presentando alla vendita gli stessi

15 Le considerazioni appena esposte nel testo non devono indurre il lettore a ritenere il profitto e la sua massimizzazione come l'obiettivo strategico dell'azienda; tuttavia, conveniamo nel ritenere che la sua constatazione, al termine di un qualunque periodo amministrativo, è indubbiamente necessaria, poiché, fra l'altro, assume il significato gestionale evidenziato nel testo, pur non volendone esaltare la "misura" che risulta troppo spesso dipendente perfino dai procedimenti computistici adottati nel rilevarla. Ribadiamo, pertanto, che la funzione dell'azienda è la "creazione di valore" di cui, semmai, il profitto è componente. 
prodotti o servizi. Nella prima ipotesi operativa il risultato economico conferma l'esistenza di un'apprezzabile sintonia e di una felice coordinazione fra unità e molteplicità, cioè fra azienda ed ambiente; nel secondo caso, invece, il tessuto socio-economico tende a rigettare una cellula, l'azienda appunto, che culturalmente risulta ad esso estranea.

Dunque, i documenti contabili sono "stringhe" di valori non solo di tipo quantitativo-monetario, ma soprattutto etico-culturale. Per questo motivo, lo studio morfologico e fisiologico dell'azienda non può fermarsi alla sola dimensione quantitativa espressa dai montanti esposti in quei documenti, ma attraverso questi dobbiamo "carotizzare" e penetrare nelle sue profondità motivazionali, fino a raggiungerne l'anima.

\section{BIBLIOGRAFIA}

CATTURI, Giuseppe (1987) "La scrittura in partita doppia come mezzo di comunicazione tra operatori economici di differenti aree territoriali". Per Lino AzzINI, Saggi di Economia Aziendale. Milano: Giuffrè, pp. 249264.

- (1995) "L'azienda ha un'anima", Summa, 94, giugno.

- (1996) "Cor magis tibi Sena pandit", Intervento di apertura dei lavori al VII Incontro annuale di Business Ethics Research Centres in Europe, Siena.

- (a cura di) (2009) I vizi aziendali - Vademecun al contrario dell'azienda di successo. Milano: Ipsoa.

DE GeUS, A. (1997) The Living Company. Boston: Harvard Business School Press.

HAECKEL, Stephan (1999) Adaptive Enterprise: Creating and Leading Senseand-Respond Organizations. Foreword by Adrian SLYWOTZKY. Boston: Harvard Business School Press.

KaUfFman, Stuart (1995) At Home in the Universe. Oxford University Press.

KaUfMANN, Massimo (2004) "Arte - domande e risposte". Nicoletta PollaMATTIOT (a cura di) Riscoprire il silenzio. Arte, musica, poesia, natura fra ascolto e comunicazione. Milano: Baldini \& Castoldi Dalai.

LOVELOCK, Jim (1992) L'ipotesi Gaia, a cura di P. BUNYARD e E. GOLDSMITH. Como: Red. 
MORGAN, Gareth (1997) Images of Organization. London: Sage.

PASCALE, R.T.; M. MILLEMANN and L. GIOIA (2000) Surfing the Edge of Chaos: The Laws of Nature and the New Laws of Business. London: Texere.

STACEY, Ralph D. (1996) Complexity and Creativity in Organizations. San Francisco: Berrett Koehler. 